\title{
A Study on the Effectiveness of Herbal Medicine Treatment for 755 Traffic Accident Patients-A Retrospective Review According to the Type of Herbal Medicine Prescription
}

\author{
Jae Ryong Bae ${ }^{1}$, Hun Mo $\mathrm{Ahn}^{1}$, Ju Hee Roh ${ }^{1}$, Min Gun Jo ${ }^{1}$ \\ Hye Ryeon $\mathrm{Kim}^{2}$, Seon Hye Kim², Won Suk Sung ${ }^{2}$, Eun Jung Kim \\ ${ }^{1}$ The Korean Academy of Medical Gi-Gong \\ ${ }^{2}$ Department of Acupuncture \& Moxibustion Medicine, College of Korean Medicine, Dongguk University
}

\begin{abstract}
Objectives: This study conducted a retrospective review to compare the effectiveness of herbal medicine, while confirming the effectiveness of Korean medicine (KM) in traffic accidents (TA).

Methods: We investigated the medical records of TA patients who were hospitalized at Muuido Korean medicine hospital from January 2016 to June 2018 retrospectively. Medical records included general characteristics (Gender, age), TA \& treatment-related information (Collision type, chief complaint, hospitalization period, and herbal medicine prescription), and clinical scales (Five-scale improvement, numerical rating scale (NRS)). Statistical analysis was performed for data distribution and effectiveness comparison.

Results: 755 TA patients showed 0.78:1 gender ratio. 20-50s years old, rear collision, pain in the cervical and lumbar spine, and hospitalization period within 14 days were the majority. KM improved $83.18 \%$ of 755 TA patients' symptoms and ROM over 'improvement' level, which indicated 30-70\% improvement. With common KM treatments including acupuncture, cupping, heat therapy, and manipulation therapy, TA patients received various herbal medicines and herbal medicine for breaking severe blood stasis (Tongdo-san) showed improvement in both five-scale improvement and NRS change at the same time.

Conclusions: We could confirm the effectiveness of KM, particularly herbal medicine in TA. It would be necessary to develop new herbal medicines such as Tongdo-san to treat TA-related symptoms.
\end{abstract}

Key Words : Korean medicine (KM), effectiveness, herbal medicine, Tongdo-san, traffic accident (TA)

\section{Introduction}

In line with the rise of traffic density, the incidence of traffic accidents (TA) has increased and 2017 TA-related medical costs increased to \#1.76 trillion KRW in South Korea. Among them, the medical costs used in Korean medicine (KM) were \#554 billion
KRW, which was a $20.59 \%$ increase compared to $2016^{1)}$.

The expected reason for the increased use of $\mathrm{KM}$ is various $\mathrm{KM}$ treatments. Based on traditional treatments such as acupuncture, moxibustion, cupping, physical therapy, and herbal medicine ${ }^{2)}$, it has been diversified with new treatments such as pharmacopuncture, chuna manipulation, and Do-in conduction exercise ${ }^{3)}$.

\footnotetext{
- Received : 23 November 2018 - Revised : 3 December 2018 Accepted : 3 December 2018

- Correspondence to : Eun Jung Kim

Department of Acupuncture \& Moxibustion Medicine, Dongguk University Bundang Oriental Hospital, 268, Buljeong-ro, Bundang-gu, Seongnam-si, Gyeonggi-do, 13601, Republic of Korea,

Tel : +82-31-710-3751, E-mail : hanijjung@naver.com
} 
These treatments have advantage of being applicable to various symptoms caused by TA.

The symptoms by TA have been reported in numerous articles. Since the Quebec Task Force proposed the classification of whiplash-associated disorders with musculoskeletal symptoms including pain, stiffness, or tenderness ${ }^{4}$, various articles have reported polymorphous symptoms including headache, muscle weakness, paresthesia, memory impairment, tinnitus ${ }^{5)}$, vertigo ${ }^{6}$ vomiting $^{7}$, dizziness, unsteadiness, and visual disturbance ${ }^{8)}$.

$\mathrm{KM}$ might be able to approach these symptoms with its unique pattern identification (PI). KM doctors diagnose TA-related symptoms and treat TA patients according to the PI. Herbal medicine is a KM treatment that depends on the PI. Many studies ${ }^{2,9)}$ have summarized and reported herbal medicines that $\mathrm{KM}$ doctors could use as treatments for TA. However, few studies have verified the effectiveness specifically according to herbal medicine.

Therefore, the purpose of this study is to investigate the general characteristics of TA patients, to confirm the effectiveness of $\mathrm{KM}$ in TA, and to compare the effectiveness according to herbal medicine. We conducted a retrospective review by using the medical records of TA patients who were hospitalized at Muuido Korean medicine hospital.

\section{Materials and Methods}

\section{Subjects}

We investigated TA patients who were hospitalized at Muuido Korean medicine hospital by TA from January 2016 to June 2018.

\section{Treatments}

\section{1) Acupuncture}

Patients received acupuncture treatment once per day with sterilized stainless steel needles $(0.20 \mathrm{~mm}$ width and $30 \mathrm{~mm}$ length, DB108C; Dongbang Medical Co., Boryung-si, South Korea and $0.16 \mathrm{~mm}$ width and 7 mm length, HL-505; Haenglim Medical Co., Yeoju-gun, South Korea) for 20 minutes.

\section{2) Cupping}

Patients received cupping treatment once per day with the retained cupping method. Treatment sites were based on the abdomen (including ST25, CV4, and CV12) and back (including bladder meridian), and added to the patient's symptoms.

\section{3) Heat therapy}

Patients received heat therapy by using hot pack $\left(40-45^{\circ} \mathrm{C}\right)$ for 10 minutes twice per day.

\section{4) Manipulation therapy}

Patients received manipulation therapy once per day by Whidam's Su-Gi therapy ${ }^{10}$. Treatment sites were based on the spine and limbs. Su-Gi therapies were offered with pressing manipulation (壓法, surrounding and pressing treatment sites with fingers) in common, and added tapping (打法, relieving treatment sites with hand's edge) or rubbing manipulation (擦法, relieving treatment sites with finger tips) according to the muscle tension.

\section{5) Herbal medicine}

Patients received herbal medicine treatment three times per day, 30 minutes after meals. The prescription was determined according to the PI that KM doctors considering the chief complaint, medical history, constitutions, and others. Commonly prescribed herbal medicines and their arrangements were as follows: 
Journal of Korean Medicine 2018;39(4)

Table 1. Arrangement of Herbal Medicine by Prescription Purpose

\begin{tabular}{|c|c|}
\hline Prescription purpose & Herbal medicine \\
\hline Others & Haedoc-dan, Jinjung-won, Hanggi-hwan \\
\hline Tonifying qi \& blood (補氣血, TQB) & Bojungikgi-tang, Guibi-tang, Sogeonjung-tang, Ssanghwa-tang \\
\hline Dispelling dampness and harmonizing stomach (祛濕安胃, DDHS) & Jungli-tang, Pyungwi-san \\
\hline Breaking mild blood stasis (破瘀-經, BBS(m)) & Dangguijakyak-san \\
\hline Breaking severe blood stasis (破瘀-重, BBS(s)) & Tongdo-san \\
\hline Dispelling wind \& cold (祛風寒, DWC) & Dusokohwa-eum, Galgeun-tang, Samso-eum, Sibsin-tang \\
\hline 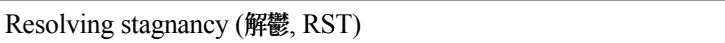 & Soshiho-tang, Soyo-san, Woohwangporyong-hwan \\
\hline
\end{tabular}

\section{Methods}

\section{1) Ethics statement}

This study was approved by electronic Institutional Review Board of Korea National Institute for Bioethics Policy on November 2018 (IRB No.:P01-201811-21-007).

\section{2) Data collection}

Based on the electronic medical records (EMR), we collected and summarized the data of TA patients who were hospitalized at Muuido Korean medicine hospital from January 2016 to June 2018 retrospectively. We collected the following information:

(1) General characteristics

(1) Gender

(2) Age

(2) TA \& treatment-related information

(1) Collision type

(2) Chief complaint

(3) Hospitalization period

(4) Herbal medicine prescription

(3) Clinical scales

(1) Five-scale improvement

(2) Numerical rating scale (NRS)

(4) Privacy protection

This study investigated demographic information (gender, age, etc.), medical history (post-TA chief complaint and other symptoms), various clinical outcomes (five-scale improvement, NRS), which were information that would be collected during routine care. We made efforts to protect personal information by maintaining confidentiality standards throughout the entire research process including data collection, analysis, writing, and publishing, by recording and distinguishing study-related documents as the subject identification code, and by keeping study materials in a place inaccessible to researchers.

\section{3) Evaluation of the effectiveness}

(1) Five-scale improvement

As in previous studies ${ }^{11-2)}$, the evaluation of improvement was categorized as follows:

(1) Complete recovery: All symptoms and range of movements (ROM) improved perfectly

(2) Excellent: All symptoms and ROM improved $>70 \%$

(3) Improvement: All symptoms and ROM improved between $30-70 \%$

(4) Mild improvement: All symptoms and ROM improved $<30 \%$

(5) Poor: All symptoms and ROM were unchanged or aggravated

(2) NRS change

NRS is one of the scales that widely used to express the patient's current pain ${ }^{13)}$. The range of NRS is from 0 (no pain) to 10 (the most severe pain imaginable). 
We investigated NRS at the time of admission and discharge.

\section{4) Statistical analysis}

All statistical analyses were performed by using SPSS version 20.0 (SPSS Inc, Chicago, IL, USA). The general characteristics, TA \& treatment-related information were analyzed by calculating descriptive statistics. Continuous variables were presented as mean \pm standard deviation (SD) and categorical variables were presented as frequencies and percentages (\%). Paired t-test was used to analyze NRS change between admission and discharge. One-way analysis of variance (ANOVA) was used to compare five-scale improvement and NRS change among three or more groups. 95\% confidence intervals were calculated, and p-value $<0.05$ was considered significant for all analyses.

\section{Results}

We identified 755 TA patients during the period and investigated EMR.

\section{General characteristics}

\section{1) Gender}

Among 755 patients, gender ratio showed 0.78:1.00 with 330 males and 425 females.

\section{2) Age}

Among 755 patients, 34 patients were under 10 years old, 18 patients were aged 10s, 101 patients were aged 20s, 151 patients were aged 30s, 147 patients were 40s, 183 patients were aged 50s, 86 patients were aged $60 \mathrm{~s}, 30$ patients were aged $70 \mathrm{~s}$, and 5 patients were aged 80 s. $20-50$ s years old were the majority $(77.09 \%)$.
Table 2. Distribution by Gender and Age

\begin{tabular}{cccc}
\hline Age (Year) & Male & Female & Total (\%) \\
\hline$\sim 09$ & 19 & 15 & $34(4.50)$ \\
\hline $10 \sim 19$ & 9 & 9 & $18(2.38)$ \\
\hline $20 \sim 29$ & 62 & 39 & $101(13.38)$ \\
\hline $30 \sim 39$ & 74 & 77 & $151(20.00)$ \\
\hline $40 \sim 49$ & 58 & 89 & $147(19.47)$ \\
\hline $50 \sim 59$ & 63 & 120 & $183(24.24)$ \\
\hline $60 \sim 69$ & 33 & 53 & $86(11.39)$ \\
\hline $70 \sim 79$ & 11 & 19 & $30(3.97)$ \\
\hline $80 \sim$ & 1 & 4 & $5(0.66)$ \\
\hline Total & 330 & 425 & $755(100.0)$ \\
\hline & & &
\end{tabular}

\section{TA \& treatment-related information}

\section{1) Collision type}

Among 755 patients, 322 patients were collided by rear $(42.65 \%), 185$ patients were collided by side (24.50\%), 111 patients were collided by frontal (14.70\%), 73 patients were collided by multiple $(9.67 \%)$, and 64 patients were others $(8.47 \%)$.

Table 3. Distribution by Collision Type

\begin{tabular}{cccc}
\hline Collision type & Male & Female & Total (\%) \\
\hline Frontal & 49 & 62 & $111(14.70)$ \\
\hline Rear & 134 & 188 & $322(42.65)$ \\
\hline Multiple & 36 & 37 & $73(9.67)$ \\
\hline Side & 79 & 106 & $185(24.50)$ \\
\hline Pedestrian & 14 & 25 & $39(5.17)$ \\
\hline Bus & 1 & 4 & $5(0.66)$ \\
\hline Bicycle & 5 & 1 & $6(0.79)$ \\
\hline Motorcycle & 12 & 2 & $14(1.85)$ \\
\hline Total & 330 & 425 & $755(100.0)$ \\
\hline
\end{tabular}

\section{2) Chief complaint}

The majority of the 755 patients complained the pain in the cervical $(74.57 \%)$ and lumbar $(71.52 \%)$ spine. More than $30 \%$ of patients complained the pain in the shoulder (45.96\%) and headache (32.19\%). Other chief complaints such as knee (14.57\%), back (14.30\%), chest $(10.86 \%)$, lower limb $(10.60 \%)$, upper limb 
(10.20\%), wrist $(10.07 \%)$, ankle (7.42\%), insomnia (4.77\%), elbow (2.78\%), and abdomen (1.59\%) pain also were checked.

Table 4. Distribution by Chief Complaint

\begin{tabular}{cccc}
\hline $\begin{array}{c}\text { Part of chief } \\
\text { complaint }\end{array}$ & Male & Female & Total (\%) \\
\hline Cervical & 244 & 319 & $563(74.57)$ \\
\hline Lumbar & 238 & 302 & $540(71.52)$ \\
\hline Shoulder & 132 & 215 & $347(45.96)$ \\
\hline Back & 40 & 68 & $108(14.30)$ \\
\hline Upper limb & 28 & 49 & $77(10.20)$ \\
\hline Elbow & 10 & 11 & $21(2.78)$ \\
\hline Wrist & 29 & 47 & $76(10.07)$ \\
\hline Lower limb & 37 & 43 & $80(10.60)$ \\
\hline Knee & 51 & 59 & $110(14.57)$ \\
\hline Ankle & 17 & 39 & $56(7.42)$ \\
\hline Headache & 87 & 156 & $243(32.19)$ \\
\hline Chest & 27 & 55 & $82(10.86)$ \\
\hline Abdomen & 0 & 12 & $12(1.59)$ \\
\hline Insomnia & 16 & 20 & $36(4.77)$ \\
\hline
\end{tabular}

\section{3) Hospitalization period}

The majority of 755 patients were hospitalized within 14 days $(91.65 \%) .314$ patients were hospitalized for 8-14 days (41.59\%), 312 patients were hospitalized for 4-7 days (41.32\%), and 66 patients were hospitalized within 3 days $(8.74 \%)$.

Table 5. Distribution by Hospitalization Period

\begin{tabular}{cccc}
\hline $\begin{array}{c}\text { Hospitalization } \\
\text { period (Day) }\end{array}$ & Male & Female & Total (\%) \\
\hline$\leq 03$ & 42 & 24 & $66(8.74)$ \\
\hline $04 \sim 07$ & 151 & 161 & $312(41.32)$ \\
\hline $08 \sim 14$ & 118 & 196 & $314(41.59)$ \\
\hline $15 \sim 21$ & 10 & 33 & $43(5.70)$ \\
\hline $22 \sim 28$ & 5 & 2 & $7(0.93)$ \\
\hline$\geq 29$ & 4 & 9 & $13(1.72)$ \\
\hline Total & 330 & 425 & $755(100.0)$ \\
\hline
\end{tabular}

\section{4) Herbal medicine prescription}

Herbal medicine prescription was based on the first used or the most used herbal medicine during the hospitalization. The majority of 755 patients received herbal medicine for breaking blood stasis $(83.70 \%)$ and 2/3 among them received herbal medicine for breaking severe blood stasis. Other herbal medicine for Dispelling dampness and harmonizing stomach (8.48\%), resolving stagnancy $(4.50 \%)$, dispelling wind \& cold (1.06\%), and tonifying qi \& blood (0.66\%) followed.

Table 6. Distribution by Herbal Medicine Prescription

\begin{tabular}{cccc}
\hline Herbal medicine & Male & Female & Total (\%) \\
\hline Others & 6 & 6 & $12(1.59)$ \\
\hline TQB & 1 & 4 & $5(0.66)$ \\
\hline DDHS & 20 & 44 & $64(8.48)$ \\
\hline BBS(m) & 67 & 139 & $206(27.28)$ \\
\hline BBS(s) & 216 & 210 & $426(56.42)$ \\
\hline DWC & 3 & 5 & $8(1.06)$ \\
\hline RST & 17 & 17 & $34(4.50)$ \\
\hline Total & 330 & 425 & $755(100.0)$ \\
\hline
\end{tabular}

$\mathrm{TQB}=$ Tonifying qi \& blood; DDHS= Dispelling dampness and harmonizing stomach; $\mathrm{BBS}(\mathrm{m})=$ Breaking mild blood stasis; BBS(s)=Breaking severe blood stasis; DWC $=$ Dispelling wind \& cold; RST=Resolving stagnancy

\section{Evaluation of the effectiveness}

\section{1) Five-scale improvement}

We distributed five-scale improvement according to the gender, collision type, and hospitalization period. Over $3 / 4$ of the TA patients $(83.18 \%)$ improved their symptoms and ROM over 'Improvement' level, which indicated $30-70 \%$ improvement. There was no clear difference in the collision type, but there was a tendency that TA patients with 4-21 hospitalization period had more probability to show their improvement over 'improvement' level $(>80.00 \%)$ than others. 
A Study on the Effectiveness of Herbal Medicine Treatment for 755 Traffic Accident Patients-A Retrospective Review According to the Type of Herbal Medicine Prescription

Table 7-1. Evaluation of the KM Effectiveness by Five-scale Improvement

\begin{tabular}{|c|c|c|c|c|c|c|c|c|c|c|c|c|c|}
\hline \multirow{2}{*}{ Factor } & \multicolumn{2}{|c|}{ Gender } & \multicolumn{5}{|c|}{ Collision type } & \multicolumn{5}{|c|}{ Hospitalization period (Day) } & \multirow{2}{*}{ Total $(\%)$} \\
\hline & Ma & $\mathrm{Fe}$ & $\mathrm{F}$ & $\mathrm{R}$ & M & $\mathrm{S}$ & $\mathrm{O}$ & $\leq 3$ & $4 \sim 7$ & $8 \sim 14$ & $15 \sim 21$ & $\geq 22$ & \\
\hline CR & 2 & 1 & 1 & 0 & 1 & 0 & 1 & 0 & 1 & 2 & 0 & 0 & $3(0.40)$ \\
\hline EX & 105 & 100 & 29 & 84 & 21 & 51 & 20 & 11 & 78 & 103 & 7 & 6 & $205(27.15)$ \\
\hline IM & 174 & 246 & 65 & 177 & 41 & 101 & 36 & 29 & 172 & 179 & 31 & 9 & $420(55.63)$ \\
\hline MI & 44 & 76 & 16 & 58 & 10 & 30 & 6 & 20 & 60 & 30 & 5 & 5 & $120(15.89)$ \\
\hline $\mathrm{PO}$ & 5 & 2 & 0 & 3 & 0 & 3 & 1 & 6 & 1 & 0 & 0 & 0 & $7(0.93)$ \\
\hline Total & 330 & 425 & 111 & 322 & 73 & 185 & 64 & 66 & 312 & 314 & 43 & 20 & $755(100.0)$ \\
\hline $\begin{array}{c}\text { Probability } \\
\text { to show } \geq \mathrm{IM}(\%)\end{array}$ & 85.15 & 81.64 & 85.58 & 81.05 & 86.30 & 82.16 & 89.06 & 60.60 & 80.44 & 90.44 & 88.37 & 75.00 & \\
\hline
\end{tabular}

$\mathrm{CR}=$ Complete recovery; $\mathrm{EX}=$ Excellent; $\mathrm{IM}=$ Improvement; $\mathrm{MI}=$ Mild improvement; $\mathrm{PO}=\mathrm{Poor}$;

$\mathrm{Ma}=$ Male; Fe=Female; $\mathrm{F}=$ Frontal; $\mathrm{R}=$ Rear; $\mathrm{M}=$ Multiple; $\mathrm{S}=$ Side; $\mathrm{O}=$ Others

Table 7-2. Evaluation of the Herbal Medicine Effectiveness by Five-scale Improvement

\begin{tabular}{|c|c|c|c|c|c|c|c|}
\hline \multirow{2}{*}{ Herbal medicine } & \multicolumn{5}{|c|}{ Five-scale improvement } & \multirow{2}{*}{ Total } & \multirow{2}{*}{$\begin{array}{c}\text { Five-scale } \\
\text { score average }\end{array}$} \\
\hline & $\mathrm{CR}$ & EX & IM & MI & $\mathrm{PO}$ & & \\
\hline Others & 0 & 3 & 8 & 1 & 0 & 12 & $2.833 \pm 0.577$ \\
\hline TQB & 0 & 2 & 2 & 1 & 0 & 5 & $2.800 \pm 0.836$ \\
\hline DDHS & 0 & 14 & 36 & 14 & 0 & 64 & $3.000 \pm 0.666$ \\
\hline $\mathrm{BBS}(\mathrm{m})$ & 2 & 54 & 105 & 43 & 2 & 206 & $2.946 \pm 0.740$ \\
\hline BBS(s) & 1 & 113 & 249 & 58 & 5 & 426 & $2.889 \pm 0.668$ \\
\hline DWC & 0 & 1 & 6 & 1 & 0 & 8 & $3.000 \pm 0.534$ \\
\hline RST & 0 & 18 & 14 & 2 & 0 & 34 & $2.529 \pm 0.614$ \\
\hline p-value & & & & & & & $.049 *$ \\
\hline Total & 3 & 205 & 420 & 120 & 7 & 755 & $2.898 \pm 0.688$ \\
\hline
\end{tabular}

Score average was presented as mean \pm SD

* By One-way analysis of variance (ANOVA)

$\mathrm{CR}=$ Complete recovery; $\mathrm{EX}=$ Excellent; $\mathrm{IM}=$ Improvement; $\mathrm{MI}=$ Mild improvement; $\mathrm{PO}=\mathrm{Poor}$; $\mathrm{TQB}=$ Tonifying qi \& blood; $\mathrm{DDHS}=$ Dispelling dampness and harmonizing stomach; $\mathrm{BBS}(\mathrm{m})=$ Breaking mild blood stasis; $\mathrm{BBS}(\mathrm{s})=$ Breaking severe blood stasis; DWC=Dispelling wind \& cold; RST=Resolving stagnancy

We scored 1 point for 'Complete recovery', 2 points for 'Excellent', 3 points for 'Improvement', 4 points for 'Mild improvement', 5 points for 'Poor' level, and then we calculated the average and evaluated the effectiveness. The average of TA patients' five-scale improvement also showed similar results. The average of 755 TA patients' five-scale improvement showed 2.898 which meant better than 'Improvement' level. According to the herbal medicine, herbal medicine for resolving stagnancy, tonifying qi \& blood, breaking severe blood stasis showed better improvement than total average. In the comparison among herbal medicines, there was a significant difference $(p<0.05)$.

\section{2) NRS change}

We investigated NRS change between admission and discharge in the symptoms that more than 100 patients complained. As a result, the pain in the cervical and lumbar spine, shoulder, headache, knee, and back improved significantly $(\mathrm{p}<0.05)$. According to the herbal medicine, herbal medicine for dispelling dampness and harmonizing stomach, breaking severe blood stasis showed better improvement than total average. In the comparison among herbal medicines, there was a significant difference in cervical and lumbar spine, and headache $(\mathrm{p}<0.05)$. 
Table 8-1. Evaluation of the Herbal medicine Effectiveness by NRS Change (1)

\begin{tabular}{|c|c|c|c|c|c|c|c|c|c|c|c|c|c|c|c|}
\hline \multirow{2}{*}{$\begin{array}{c}\text { Herbal } \\
\text { medicine }\end{array}$} & \multicolumn{5}{|c|}{ Cervical } & \multicolumn{5}{|c|}{ Lumbar } & \multicolumn{5}{|c|}{ Shoulder } \\
\hline & $\mathrm{n}$ & Adm. & Dis. & Diff. & $\mathrm{p}$ & $\mathrm{n}$ & Adm. & Dis. & Diff. & $\mathrm{p}$ & $\mathrm{n}$ & Adm. & Dis. & Diff. & $\mathrm{p}$ \\
\hline Others & 11 & $\begin{array}{c}7.909 \\
\pm 1.868\end{array}$ & $\begin{array}{c}3.363 \\
\pm 1.120\end{array}$ & $\begin{array}{c}4.545 \\
\pm 1.694\end{array}$ & $.000 \dagger$ & 11 & $\begin{array}{c}7.636 \\
\pm 2.062\end{array}$ & $\begin{array}{c}3.545 \\
\pm 1.035\end{array}$ & $\begin{array}{c}4.090 \\
\pm 2.119\end{array}$ & $.000 \dagger$ & 7 & $\begin{array}{c}8.428 \\
\pm 1.511\end{array}$ & $\begin{array}{c}3.142 \\
\pm 0.690\end{array}$ & $\begin{array}{c}5.285 \\
\pm 1.112\end{array}$ & $.000 \dagger$ \\
\hline TQB & 4 & $\begin{array}{c}6.250 \\
\pm 2.500\end{array}$ & $\begin{array}{c}3.250 \\
\pm 1.707\end{array}$ & $\begin{array}{c}3.000 \\
\pm 1.825\end{array}$ & $.046 †$ & 2 & $\begin{array}{c}6.000 \\
\pm .0000\end{array}$ & $\begin{array}{c}2.500 \\
\pm 0.707\end{array}$ & $\begin{array}{c}3.500 \\
\pm 0.707\end{array}$ & $.090 \dagger$ & 2 & $\begin{array}{c}5.000 \\
\pm 0.000\end{array}$ & $\begin{array}{c}2.500 \\
\pm 2.121\end{array}$ & $\begin{array}{c}2.500 \\
\pm 2.121\end{array}$ & .344 \\
\hline DDHS & 42 & $\begin{array}{c}6.881 \\
\pm 1.670\end{array}$ & $\begin{array}{c}3.523 \\
\pm 1.347\end{array}$ & $\begin{array}{c}3.357 \\
\pm 1.428\end{array}$ & $.000 \dagger$ & 42 & $\begin{array}{c}6.976 \\
\pm 1.703\end{array}$ & $\begin{array}{c}3.523 \\
\pm 1.469\end{array}$ & $\begin{array}{c}3.452 \\
\pm 1.213\end{array}$ & $.000 \dagger$ & 25 & $\begin{array}{c}7.440 \\
\pm 1.583\end{array}$ & $\begin{array}{c}3.800 \\
\pm 1.414\end{array}$ & $\begin{array}{c}3.640 \\
\pm 1.410\end{array}$ & $.000 \dagger$ \\
\hline $\mathrm{BBS}(\mathrm{m})$ & 155 & $\begin{array}{c}7.071 \\
\pm 1.769\end{array}$ & $\begin{array}{c}3.548 \\
\pm 1.567\end{array}$ & $\begin{array}{c}3.522 \\
\pm 1.465\end{array}$ & $.000 \dagger$ & 134 & $\begin{array}{c}6.776 \\
\pm 1.670\end{array}$ & $\begin{array}{c}3.432 \\
\pm 1.427\end{array}$ & $\begin{array}{c}3.343 \\
\pm 1.608\end{array}$ & $.000 \dagger$ & 117 & $\begin{array}{c}7.034 \\
\pm 1.686\end{array}$ & $\begin{array}{c}3.589 \\
\pm 1.565\end{array}$ & $\begin{array}{c}3.444 \\
\pm 1.583\end{array}$ & $.000 \dagger$ \\
\hline BBS(s) & 328 & $\begin{array}{c}7.344 \\
\pm 1.664\end{array}$ & $\begin{array}{c}3.417 \\
\pm 1.331\end{array}$ & $\begin{array}{c}3.926 \\
\pm 1.566\end{array}$ & $.000 \dagger$ & 332 & $\begin{array}{c}7.500 \\
\pm 1.691\end{array}$ & $\begin{array}{c}3.524 \\
\pm 1.453\end{array}$ & $\begin{array}{c}3.975 \\
\pm 1.568\end{array}$ & $.000 \dagger$ & 185 & $\begin{array}{c}7.448 \\
\pm 1.690\end{array}$ & $\begin{array}{c}3.432 \\
\pm 1.317\end{array}$ & $\begin{array}{c}4.016 \\
\pm 1.558\end{array}$ & $.000 \dagger$ \\
\hline DWC & 8 & $\begin{array}{c}8.250 \\
\pm 1.832\end{array}$ & $\begin{array}{c}3.875 \\
\pm 0.991\end{array}$ & $\begin{array}{c}4.375 \\
\pm 1.597\end{array}$ & .487 & 5 & $\begin{array}{c}8.200 \\
\pm 1.788\end{array}$ & $\begin{array}{c}3.800 \\
\pm 1.483\end{array}$ & $\begin{array}{c}4.400 \\
\pm 1.516\end{array}$ & $.003 \dagger$ & 4 & $\begin{array}{c}8.500 \\
\pm 1.732\end{array}$ & $\begin{array}{c}4.000 \\
\pm 0.816\end{array}$ & $\begin{array}{c}4.500 \\
\pm 1.732\end{array}$ & $.014 \dagger$ \\
\hline RST & 15 & $\begin{array}{c}6.800 \\
\pm 1.567\end{array}$ & $\begin{array}{c}3.266 \\
\pm 1.334\end{array}$ & $\begin{array}{c}3.533 \\
\pm 1.641\end{array}$ & $.000 \dagger$ & 14 & $\begin{array}{c}7.142 \\
\pm 1.292\end{array}$ & $\begin{array}{c}3.500 \\
\pm 1.400\end{array}$ & $\begin{array}{c}3.642 \\
\pm 1.215\end{array}$ & $.000 \dagger$ & 7 & $\begin{array}{c}5.571 \\
\pm 0.534\end{array}$ & $\begin{array}{c}2.857 \\
\pm 0.899\end{array}$ & $\begin{array}{c}2.714 \\
\pm 0.951\end{array}$ & $.000 \dagger$ \\
\hline p-value & & & & $.015^{*}$ & & & & & $.005^{*}$ & & & & & $.001^{*}$ & \\
\hline Total & 563 & $\begin{array}{c}7.236 \\
\pm 1.712\end{array}$ & $\begin{array}{c}3.461 \\
\pm 1.392\end{array}$ & $\begin{array}{c}3.774 \\
\pm 1.548\end{array}$ & $.000 \dagger$ & 540 & $\begin{array}{c}7.274 \\
\pm 1.710\end{array}$ & $\begin{array}{c}3.500 \\
\pm 1.433\end{array}$ & $\begin{array}{c}3.774 \\
\pm 1.575\end{array}$ & $.000 \dagger$ & 347 & $\begin{array}{c}7.288 \\
\pm 1.699\end{array}$ & $\begin{array}{c}3.495 \\
\pm 1.398\end{array}$ & $\begin{array}{c}3.792 \\
\pm 1.581\end{array}$ & $.000 \dagger$ \\
\hline
\end{tabular}

Values were presented as mean $\pm \mathrm{SD}$, † By paired t-test, * By One-way analysis of variance (ANOVA)

$\mathrm{TQB}=$ Tonifying qi \& blood; DDHS= Dispelling dampness and harmonizing stomach; $\mathrm{BBS}(\mathrm{m})=$ Breaking mild blood stasis; $\mathrm{BBS}(\mathrm{s})=$ Breaking severe blood stasis; DWC $=$ Dispelling wind \& cold; RST=Resolving stagnancy; Adm. $=$ Admission; Dis. $=$ Discharge; Diff.=Difference

Table 8-2. Evaluation of the Herbal Medicine Effectiveness by NRS Change (2)

\begin{tabular}{|c|c|c|c|c|c|c|c|c|c|c|c|c|c|c|c|}
\hline \multirow{2}{*}{$\begin{array}{c}\text { Herbal } \\
\text { medicine }\end{array}$} & \multicolumn{5}{|c|}{ Headache } & \multicolumn{5}{|c|}{ Knee } & \multicolumn{5}{|c|}{ Back } \\
\hline & $\mathrm{n}$ & Adm. & Dis. & Diff. & $\mathrm{p}$ & $\mathrm{n}$ & Adm. & Dis. & Diff. & $\mathrm{p}$ & $\mathrm{n}$ & Adm. & Dis. & Diff. & $\mathrm{p}$ \\
\hline Others & 5 & $\begin{array}{c}8.600 \\
\pm 0.894\end{array}$ & $\begin{array}{c}3.200 \\
\pm 1.095\end{array}$ & $\begin{array}{c}5.400 \\
\pm 0.894\end{array}$ & $.000 \dagger$ & 0 & - & - & - & - & 2 & $\begin{array}{c}5.500 \\
\pm 0.707\end{array}$ & $\begin{array}{c}3.000 \\
\pm 0.000\end{array}$ & $\begin{array}{c}2.500 \\
\pm 0.707\end{array}$ & .126 \\
\hline TQB & 3 & $\begin{array}{c}5.333 \\
\pm 3.511\end{array}$ & $\begin{array}{c}3.666 \\
\pm 2.081\end{array}$ & $\begin{array}{c}1.666 \\
\pm 1.527\end{array}$ & .199 & 1 & $\begin{array}{c}5.000 \\
\pm 0.000\end{array}$ & $\begin{array}{c}3.000 \\
\pm 0.000\end{array}$ & $\begin{array}{c}2.000 \\
\pm 0.000\end{array}$ & - & 1 & $\begin{array}{c}5.000 \\
\pm 0.000\end{array}$ & $\begin{array}{c}3.000 \\
\pm 0.000\end{array}$ & $\begin{array}{c}2.000 \\
\pm 0.000\end{array}$ & - \\
\hline DDHS & 30 & $\begin{array}{c}7.133 \\
\pm 2.330 \\
\end{array}$ & $\begin{array}{c}3.966 \\
\pm 1.245 \\
\end{array}$ & $\begin{array}{c}3.166 \\
\pm 1.931 \\
\end{array}$ & $.000 \dagger$ & 5 & $\begin{array}{c}7.600 \\
\pm 1.673 \\
\end{array}$ & $\begin{array}{c}3.400 \\
\pm 1.673 \\
\end{array}$ & $\begin{array}{c}4.200 \\
\pm 0.447 \\
\end{array}$ & $.000 \dagger$ & 14 & $\begin{array}{c}7.642 \\
\pm 2.023 \\
\end{array}$ & $\begin{array}{c}3.571 \\
\pm 1.283 \\
\end{array}$ & $\begin{array}{c}4.071 \\
\pm 1.591 \\
\end{array}$ & $.000 \dagger$ \\
\hline $\mathrm{BBS}(\mathrm{m})$ & 70 & $\begin{array}{c}6.814 \\
\pm 2.168\end{array}$ & $\begin{array}{c}3.400 \\
\pm 1.376\end{array}$ & $\begin{array}{c}3.414 \\
\pm 1.907\end{array}$ & $.000 \dagger$ & 36 & $\begin{array}{c}6.777 \\
\pm 1.914\end{array}$ & $\begin{array}{c}3.333 \\
\pm 1.454\end{array}$ & $\begin{array}{c}3.444 \\
\pm 1.646\end{array}$ & $.000 \dagger$ & 25 & $\begin{array}{c}6.880 \\
\pm 1.508\end{array}$ & $\begin{array}{c}3.360 \\
\pm 1.186\end{array}$ & $\begin{array}{c}3.520 \\
\pm 1.610\end{array}$ & $.000 \dagger$ \\
\hline BBS(s) & 121 & $\begin{array}{c}6.991 \\
\pm 2.018\end{array}$ & $\begin{array}{c}3.148 \\
\pm 1.314\end{array}$ & $\begin{array}{c}3.843 \\
\pm 1.746\end{array}$ & $.000 \dagger$ & 66 & $\begin{array}{c}6.727 \\
\pm 1.917\end{array}$ & $\begin{array}{c}3.272 \\
\pm 1.222\end{array}$ & $\begin{array}{c}3.454 \\
\pm 1.738\end{array}$ & $.000 \dagger$ & 59 & $\begin{array}{c}7.474 \\
\pm 1.633\end{array}$ & $\begin{array}{c}3.474 \\
\pm 1.394\end{array}$ & $\begin{array}{c}4.000 \\
\pm 1.629\end{array}$ & $.000 \dagger$ \\
\hline DWC & 1 & $\begin{array}{c}9.000 \\
\pm 0.000 \\
\end{array}$ & $\begin{array}{c}6.000 \\
\pm 0.000 \\
\end{array}$ & $\begin{array}{c}3.000 \\
\pm 0.000 \\
\end{array}$ & - & 1 & $\begin{array}{c}8.000 \\
\pm 0.000 \\
\end{array}$ & $\begin{array}{c}4.000 \\
\pm 0.000 \\
\end{array}$ & $\begin{array}{c}4.000 \\
\pm 0.000 \\
\end{array}$ & - & 3 & $\begin{array}{c}8.666 \\
\pm 1.154 \\
\end{array}$ & $\begin{array}{c}3.666 \\
\pm 2.081 \\
\end{array}$ & $\begin{array}{c}5.000 \\
\pm 1.000 \\
\end{array}$ & $.013 \dagger$ \\
\hline RST & 13 & $\begin{array}{c}7.769 \\
\pm 1.690 \\
\end{array}$ & $\begin{array}{c}3.615 \\
\pm 1.325 \\
\end{array}$ & $\begin{array}{c}4.153 \\
\pm 1.463 \\
\end{array}$ & $.000 \dagger$ & 1 & $\begin{array}{c}7.000 \\
\pm 0.000 \\
\end{array}$ & $\begin{array}{c}3.000 \\
\pm 0.000 \\
\end{array}$ & $\begin{array}{c}4.000 \\
\pm 0.000 \\
\end{array}$ & - & 4 & $\begin{array}{c}7.250 \\
\pm 1.258 \\
\end{array}$ & $\begin{array}{c}3.250 \\
\pm 1.258 \\
\end{array}$ & $\begin{array}{c}4.000 \\
\pm 0.816 \\
\end{array}$ & $.002 \dagger$ \\
\hline $\mathrm{p}$-value & & & & $.016^{*}$ & & & & & .622 & & & & & .439 & \\
\hline Total & 243 & $\begin{array}{c}7.020 \\
\pm 2.101\end{array}$ & $\begin{array}{c}3.366 \\
\pm 1.352\end{array}$ & $\begin{array}{c}3.654 \\
\pm 1.823\end{array}$ & $.000 \dagger$ & 110 & $\begin{array}{c}6.781 \\
\pm 1.883\end{array}$ & $\begin{array}{c}3.300 \\
\pm 1.296\end{array}$ & $\begin{array}{c}3.481 \\
\pm 1.651\end{array}$ & $.000 \dagger$ & 108 & $\begin{array}{c}7.324 \\
\pm 1.662\end{array}$ & $\begin{array}{c}3.444 \\
\pm 1.306\end{array}$ & $\begin{array}{c}3.879 \\
\pm 1.586\end{array}$ & $.000 \dagger$ \\
\hline
\end{tabular}

Values were presented as mean $\pm \mathrm{SD}, \dagger$ By paired t-test, $*$ By One-way analysis of variance (ANOVA)

$\mathrm{TQB}=$ Tonifying qi \& blood; DDHS= Dispelling dampness and harmonizing stomach; $\mathrm{BBS}(\mathrm{m})=$ Breaking mild blood stasis; $\mathrm{BBS}(\mathrm{s})=$ Breaking severe blood stasis; DWC=Dispelling wind \& cold; RST=Resolving stagnancy; Adm. $=$ Admission; Dis. $=$ Discharge; Diff.=Difference 
Table 8-3. Evaluation of the Herbal Medicine Effectiveness by NRS Change (3)

\begin{tabular}{|c|c|c|c|c|c|c|c|c|c|c|c|c|c|c|c|c|}
\hline \multirow{2}{*}{$\begin{array}{c}\text { Herbal } \\
\text { medicine }\end{array}$} & \multicolumn{2}{|c|}{ Chest } & \multicolumn{2}{|c|}{ Lower limb } & \multicolumn{2}{|c|}{ Upper limb } & \multicolumn{2}{|c|}{ Wrist } & \multicolumn{2}{|c|}{ Ankle } & \multicolumn{2}{|c|}{ Insomnia } & \multicolumn{2}{|c|}{ Elbow } & \multicolumn{2}{|c|}{ Abdomen } \\
\hline & $\mathrm{n}$ & Diff. & $\mathrm{n}$ & Diff. & $\mathrm{n}$ & Diff. & $\mathrm{n}$ & Diff. & $\mathrm{n}$ & Diff. & $\mathrm{n}$ & Diff. & $\mathrm{n}$ & Diff. & $\mathrm{n}$ & Diff. \\
\hline Others & 1 & $\begin{array}{c}5.000 \\
\pm 0.000\end{array}$ & 0 & - & 1 & $\begin{array}{c}6.000 \\
\pm 0.000\end{array}$ & 1 & $\begin{array}{c}6.000 \\
\pm 0.000\end{array}$ & 0 & - & 0 & - & 0 & - & 0 & - \\
\hline TQB & 1 & $\begin{array}{c}4.000 \\
\pm 0.000\end{array}$ & 0 & - & 0 & - & 0 & - & 1 & $\begin{array}{c}3.000 \\
\pm 0.000\end{array}$ & 1 & $\begin{array}{c}8.000 \\
\pm 0.000\end{array}$ & 0 & - & 0 & - \\
\hline DDHS & 9 & $\begin{array}{c}3.777 \\
\pm 1.133\end{array}$ & 2 & $\begin{array}{c}3.500 \\
\pm 0.500\end{array}$ & 9 & $\begin{array}{c}4.333 \\
\pm 0.942\end{array}$ & 7 & $\begin{array}{c}3.857 \\
\pm 1.245\end{array}$ & 1 & $\begin{array}{c}0.000 \\
\pm 0.000\end{array}$ & 9 & $\begin{array}{c}4.666 \\
\pm 1.490\end{array}$ & 0 & - & 1 & $\begin{array}{c}3.000 \\
\pm 0.000\end{array}$ \\
\hline BBS(m) & 24 & $\begin{array}{c}3.625 \\
\pm 1.494\end{array}$ & 21 & $\begin{array}{c}3.666 \\
\pm 1.458\end{array}$ & 22 & $\begin{array}{c}3.454 \\
\pm 1.558\end{array}$ & 20 & $\begin{array}{c}3.600 \\
\pm 1.496\end{array}$ & 19 & $\begin{array}{c}3.211 \\
\pm 1.471\end{array}$ & 4 & $\begin{array}{c}4.750 \\
\pm 0.433\end{array}$ & 6 & $\begin{array}{c}2.000 \\
\pm 1.632\end{array}$ & 5 & $\begin{array}{c}4.600 \\
\pm 0.489\end{array}$ \\
\hline BBS(s) & 44 & $\begin{array}{c}3.863 \\
\pm 1.673\end{array}$ & 57 & $\begin{array}{c}3.807 \\
\pm 1.626\end{array}$ & 43 & $\begin{array}{c}4.116 \\
\pm 1.512\end{array}$ & 46 & $\begin{array}{c}3.478 \\
\pm 1.896\end{array}$ & 32 & $\begin{array}{c}3.468 \\
\pm 1.767\end{array}$ & 9 & $\begin{array}{c}5.000 \\
\pm 1.632\end{array}$ & 15 & $\begin{array}{c}3.333 \\
\pm 1.813\end{array}$ & 6 & $\begin{array}{r}4.000 \\
\pm 1.632\end{array}$ \\
\hline DWC & 1 & $\begin{array}{c}6.000 \\
\pm 0.000\end{array}$ & 0 & - & 1 & $\begin{array}{c}5.000 \\
\pm 0.000\end{array}$ & 1 & $\begin{array}{c}5.000 \\
\pm 0.000\end{array}$ & 1 & $\begin{array}{c}3.000 \\
\pm 0.000\end{array}$ & 0 & - & 0 & - & 0 & - \\
\hline RST & 2 & $\begin{array}{c}3.000 \\
\pm 1.000\end{array}$ & 0 & - & 1 & $\begin{array}{c}6.000 \\
\pm 0.000\end{array}$ & 1 & $\begin{array}{c}5.000 \\
\pm 0.000\end{array}$ & 2 & $\begin{array}{c}4.000 \\
\pm 1.000\end{array}$ & 13 & $\begin{array}{c}5.000 \\
\pm 1.921\end{array}$ & 0 & - & 0 & - \\
\hline Total & 82 & $\begin{array}{c}3.805 \\
\pm 1.557\end{array}$ & 80 & $\begin{array}{c}3.763 \\
\pm 1.567\end{array}$ & 77 & $\begin{array}{c}4.013 \\
\pm 1.516\end{array}$ & 76 & $\begin{array}{c}3.618 \\
\pm 1.746\end{array}$ & 56 & $\begin{array}{c}3.321 \\
\pm 1.670\end{array}$ & 36 & $\begin{array}{c}4.972 \\
\pm 1.691\end{array}$ & 21 & $\begin{array}{c}2.952 \\
\pm 1.863\end{array}$ & 12 & $\begin{array}{c}4.167 \\
\pm 1.280\end{array}$ \\
\hline
\end{tabular}

Values were presented as mean $\pm \mathrm{SD}$

TQB=Tonifying qi \& blood; DDHS= Dispelling dampness and harmonizing stomach; $\mathrm{BBS}(\mathrm{m})=$ Breaking mild blood stasis; BBS(s)=Breaking severe blood stasis; DWC=Dispelling wind \& cold; RST=Resolving stagnancy; Diff.=Difference between admission and discharge

\section{Discussion}

In treatment for TA, the proportion of $\mathrm{KM}$ has increased. KM expenses in the automobile insurance exceeded 463 billion KRW in 2016, growing at about $37 \%$ per year for 2014-2016 ${ }^{14)}$. This tendency might be related to the results of Park et al study ${ }^{15)}$ on the high use of KM in pain and Kim et al study ${ }^{16)}$ on the high satisfaction of KM in TA, and the answer to Seo et al proposal ${ }^{17)}$ that emphasized the need to expand KM service.

In the Kim et al study ${ }^{16}$, we could also confirm the reason that TA patients changed from Western medicine to KM. Most of them chose "Get more treatments than Western medicine treatments" which would suggest various symptoms due to TA. TA-related symptoms have been reported in domestic and international studies. Park et $\mathrm{al}^{18)}$ summarized TA-related symptoms into whole-body systemic symptoms (pain, fatigue) and neurological symptoms (insomnia, anxiety) and Tanaka et $\mathrm{al}^{19)}$ suggested neurological symptoms, CSF hypovolemia, and fibromyalgia in addition to the existing Quebec classification on whiplash-associated disorders. These reports would provide a key to treatment choice.

In KM, the symptoms by TA have been considered in various pathogenesis. Park et $\mathrm{al}^{20)}$ reported that static blood, qi-blood, visceral, cold-heat PI were used to diagnose TA injury. The prescription of herbal medicine might also be variable and several studies ${ }^{21-2)}$ have classified and reported the use of herbal medicine in TA. However, few studies have verified the effectiveness of herbal medicine specifically. Song et $\mathrm{al}^{23)}$ compared the therapeutic effects of five herbal medicine with visual analogue scale (VAS) and pain disability index (PDI), but most of them were predominantly related to pain. Therefore, this study tried to analyze the effectiveness of herbal medicine 
belonging to various fields.

The arrangement of herbal medicine was based on previous studies. Lee et $\mathrm{al}^{2)}$ and Kang et $\mathrm{al}^{11)}$ distributed herbal medication according to three purpose (qi-regulating and smoothing qi flow (理氣順氣), blood-activating and stasis-dispelling (活血祛瘀), and tonifying qi \& blood (補氣(血)) and Kim et al ${ }^{24)}$ suggested blood deficiency (血虛), qi stagnation (氣滯), blood stasis (病血), dampness (濕), dryness (燥), phlegm (痰), and five viscera (五臟) as a TA pathogenesis.

With these items, we made five themes that could arrange various herbal medicines, 1) tonifying qi \& blood (補氣血) for qi \& blood deficiency (氣血虛), 2) dispelling dampness and harmonizing stomach (祛濕安 胃) for spleen stomach (脾胃) disorder, 3) breaking blood stasis (破瘀血) for static blood (瘀血), 4) dispelling wind \& cold (祛風寒) for external contraction (外感), and 5) resolving stagnancy (解蠜) for qi stagnation (氣滯). Because of the frequent use, herbal medicine for breaking blood stasis was divided according to its severity. Under these conditions, we analyzed retrospectively the medical records of 755 TA patients who were hospitalized at Muuido Korean medicine hospital from January 2016 to June 2018, and received similar acupuncture, cupping, heat therapy, and manipulation therapy while receiving different herbal medicine.

In general characteristics, this study showed 0.78:1 gender ratio and the majority of TA patients were 20-50s years old $(77.09 \%)$. High ratio of women was similar to previous studies ${ }^{9,12)}$, but different from the statistical analysis $^{25)}$ that reported high ratio of men. There was a large difference between 20-50s years, which was the majority of age. This might be due to the high level of social activity and concern about the TA-related disorders ${ }^{9}$.

In TA \& treatment related information, rear collision (42.65\%), pain in the cervical (74.57\%) and lumbar
$(71.52 \%)$ spine, within 14 days of hospitalization (91.65\%) were the majority. High occurrence of rear collision was similar to previous studies ${ }^{3,26}$, and it might be expected that pain sites were caused by hyper flexion and extension in rear collision. Other frequent pain sites were shoulder (45.96\%) and knee (14.57\%), and it was similar to Kim et al study ${ }^{3}$ that reported the frequency of neck pain (77.2\%) low back pain (75.5\%), shoulder (46.1\%) and knee pain (16.3\%). It might be anticipated that the pain site occurrence would be expected by combining the results of recent studies and grasping the tendency of an accident. Within 14 days of hospitalization period was similar to Kim et $\mathrm{al}^{9)}(84.6 \%)$ and Shin et al study ${ }^{27)}(87.09 \%)$. However, hospitalization period tendency according to the gender was different. In this study, men had tendency to be hospitalized for less than a week (58.48\%), but the proportion of 8-14 days (46.11\%) was high in women, which suggested the need of further research.

In the effectiveness of KM, $83.18 \%$ patients showed improvement over the "improvement" level, which was better than $63.40 \%$ in a 2012 study $^{12)}$ and 2.89 average score, which was better than 3.22 in a 2013 study $^{27)}$. Improvement probability according to the hospitalization period was the best in 8-14 days, which was similar to the Shin et al study ${ }^{27)}$ that showed best score in 8-14 days among 2-21 days hospitalization. This might be helpful to determine the appropriate hospitalization period. In NRS change, six symptoms that were over $10 \%$ of 755 patients' complaint improved significantly. These results would help verifying the effectiveness of KM with other similar studies.

In the effectiveness of herbal medicine, it was remarkable that herbal medicine for breaking severe blood stasis, Tongdo-san, showed improvement in both five-scale improvement and NRS change. Since Tongdo-san was recorded at Manbyeonghoichun, it has been used for severe blood stasis or qi stagnation due 
to moderate contusion ${ }^{28)}$. Except for the Lee et al study ${ }^{29)}$ on the improvement of the cardiovascular system in the experiment and Kim et al study ${ }^{30}$ on its effectiveness for TA women patients, the study about Tongdo-san was insufficient. Including herbal medicines such as Kyejibokryong-hwan ${ }^{31)}$, several methods ${ }^{32)}$ for breaking blood stasis have been attempted to treat TA. It would be necessary to conduct further study to develop new herbal medicines for TA.

This study has significance in its attempt to compare the effectiveness of herbal medicine. However, there are some limitations. First, it was conducted at a single institution, Second, there was weak relationship between PI and herbal medicine prescription because of the characteristics of retrospective review. However, this study was conducted with a large number (755) of TA patients and identified the effectiveness of herbal medicine while other similar treatments were offered. Further study with better method might be necessary.

\section{Conclusions}

We investigated and analyzed the medical records of TA patients who were hospitalized at Muuido Korean medicine hospital from January 2016 to June 2018, and concluded the following:

1. 755 TA patients showed $0.78: 1$ gender ratio and 20-50s years old with rear collision, pain in the cervical and lumbar spine, and hospitalization period within 14 days were the majority.

2. KM improved 755 TA patients' symptoms and ROM. In five-scale improvement, KM showed improvement over 'improvement' level, which indicated $30-70 \%$ improvement. NRS change in each chief complaint also showed significant improvement.
3. According to the herbal medicine, herbal medicine for resolving stagnancy, breaking severe blood stasis, and tonifying qi \& blood showed better improvement than total average in five-scale improvement. In NRS change, herbal medicine for dispelling dampness and harmonizing stomach, breaking severe blood stasis showed better improvement than total average.

4. Herbal medicine for breaking severe blood stasis, Tongdo-san, showed meaningful improvement in both five-scale improvement and NRS change.

\section{Acknowledgement}

This study was supported by a grant of the project 'Sharing proven health technology and medicine of Korean medicine', Guideline center for Korean medicine, National Development Institute of Korean medicine (HI16C0275).

\section{References}

1. Health Insurance Review \& Assessment Service. 2017 Automobile Insurance Coverage Statistics. 2018:3,5.

2. Lee EJ, Oh MS, Song TW, Lee CW. Korean medicine treatment for Traffic Accident. J Oriental Rehabilitation Medicine. 1997;7(1):218-27.

3. Kim NH, Lee YJ, Suh CY, Lee GB, Yang KJ, Kim TG, et al. A Cross-Sectional Satisfaction Survey of 845 Traffic Accident Patients Treated with Korean Medicine. J Acupunct Res. 2018;35(2):69-74.

4. Spitzer WO, Skovron ML, Salmi LR, Cassidy JD, Duranceau J, Suissa S, et al. Scientific monograph of the Quebec Task Force on Whiplash-Associated Disorders: redefining "whiplash" and its management. Spine (Phila Pa 1976). 1995;20(8 
Suppl):1S-73S.

5. Benoist M, Rouaud JP. Whiplash: myth or reality? Joint Bone Spine. 2002;69(4):358-62.

6. Endo K, Ichimaru K, Komagata M, Yamamoto $\mathrm{K}$. Cervical vertigo and dizziness after whiplash injury. Eur Spine J. 2006;15(6):886-90.

7. Sanchis-Gimeno JA, Perez-Bermejo M, Rios L, Llido S, Bastir M, Blanco-Perez E, et al. Analysis of the relationship between the double transverse foramen and the possibility of developing clinical symptoms after whiplash. Clin Anat. 2017;30(6): 761-766.

8. Treleaven J. Dizziness, unsteadiness, visual disturbances, and postural control: implications for the transition to chronic symptoms after a whiplash trauma. Spine (Phila $\mathrm{Pa}$ 1976). 2011;36(25 Suppl):S211-7.

9. Kim EG, Lee DG, Jeong WJ, Cho HS, Bae JI, Kim SH, et al. The Clinical Study on 500 Cases with Traffic Accident Patients Admitted in Oriental Medical Hospital. J Korean Acupuncture \& Moxibustion Society. 2009;26(5):29-38.

10. Lee JH, Ahn HM, Hong SC, Lee EM. The Clinical study of Su-Gi therapy`s Effects on Bell's palsy by observing of Y-system. J of Korean Academy of Medical GI-GONG. 2015;15(1):109-136.

11. Kang JH, Jang SG, Lee H, Lee BR. The clinical study on 37 cases of whiplash injury patients which caused by traffic accident. J Korean Acupuncture \& Moxibustion Society. 2002;19(3):180-191.

12. Kook KH, Oh MS. The Analysis of 509 Admission Patients in Korean Medical Hospital due to Traffic Accident. J Oriental Rehabilitation Medicine. 2012;22(4):169-183.

13. Shim SY, Park HJ, Lee JM, Lee HS. An Overview of Pain Measurements. The Korean J of Meridian \& Acupoint. 2007;24(2):77-97.

14. Song YA, Lee SY. The present status and future direction of oriental medical services in the automobile insurance. Korea Insurance Research Institute. 2017.

15. Park JE, Kwon SM. Determinants of the Utilization of Oriental Medical Services by the Elderly. J Korean Oriental Med. 2011;32(1):97-108.

16. Kim HR, Kim SH, Lee YS, Park SH, Sung WS, Cho HS, et al. Survey on Satisfaction and Symptom Improvement of Korean Medicine Treatment in 122 Cases by Traffic Accident. J Korean Oriental Med 2018;39(3):61-72.

17. Seo YJ, Kang SH, Kim YH, Choi DB, Shin HK. Systematic Review on the Customers' Use of and Satisfaction with Oriental Medical Services. Korean Oriental Med. 2010;31(1):69-80.

18. Park SY, Lee YK, Kim JS, Lim SC, Lee BH, Jung TY, et al. Survey of Oriental Medical Care for Traffic Accident Patients with Automobile insurance; 544 Cases Report. J Korean Acupuncture \& Moxibustion Society. 2009;26(3):1-10.

19. Tanaka N, Atesok K, Nakanishi K, Kamei N, Nakamae T, Kotaka S, et al. Pathology and Treatment of Traumatic Cervical Spine Syndrome: Whiplash Injury. Adv Orthop. 2018;2018:4765050.

20. Park IH, Hwang EH, Hwang MS, Heo I, Kim BJ, Lim KT, et al. A Web-based Survey on Clinical Practice Patterns of Korean Medicine Treatment for Traffic Injuries. J Korean Medicine Rehabilitation. 2017;27(4):131-45.

21. Yoon IJ, Song TW, Oh MS. Clinical Analysis of 112 Cases of Traffic Accident Patients. J Oriental Rehabilitation Medicine. 1998;8(1):317-332.

22. Shin YS, Park JB, Kim CH, Choi JL, Kim DH. Korean Medicine approach to traffic accident victims. J Korean Acupuncture \& Moxibustion Society. 2002;19(6):1-11.

23. Song YI, Jin KS, Shin HL, Yoon IJ, Choi SH, Oh MS. The Clinical Analysis of Traffic Accident Patients Treated by Oriental Medicine. J Oriental Rehabilitation Medicine. 2005;15(2):77-89. 
24. Kim MK, Heo JE, Park SM, Choi HN, Lee IS, Kim BH, et al. Comparative Study of Normal Person and Traffic Accident Patient by DSOM. $\mathrm{J}$ physiology \& pathology in Korean Medicine. 2009;23(1):245-250.

25. Traffic Accident Analysis System. Statistical Analysis of Traffic Accidents (2017). KoROAD. 2018.

26. Jeon HJ, Kim SH, Kim JH, Lee SM, Shin HY, Lee YK, et al. The clinical study on 197 cases with patients of Neck pain caused by traffic accident. J Korean Acupuncture \& Moxibustion Society. 2010;27(2):143-154.

27. Shin JH, Oh MS. The Retrospective Analysis of 1,162 Traffic Accident Inpatients in Korean Medicine Hospital. J of Korean Medicine Rehabilitation. 2013;23(4):233-50.

28. Heo J. Donguibogam. Seoul:Namsandang. 1989.

29. Lee in, Shin SH, Moon BS. Effects of Tongdosan on the Cardiovascular System in the Experimental Animals. J Int. Korean Med. 1996;17(1):34-50.

30. Kim JH, Ahn HM. A study on the Clinical Characteristics of Injured Patient Using Tongdo-san -Focused on Traffic Accidents Cases. $\mathrm{J}$ of Korean Academy of Medical GI-GONG. 2016;16(1):101-15.

31. Jo HG, Park AR, Kee YB, Kang DH, Choi JB, Sul JU. A Clinical Trial on the Blood Stasis and Efficacy of Kyejibokryong-whan(Guizhifuling-wan) in the Patients with Motor Vehicle Accident. J Oriental Rehabilitation Medicine. 2011;21(3):43-55.

32. Jeon TD, Lee HG, Hong SY, Heo DS, Yoon IJ, Oh MS. The Effectiveness of the Herb-medication based on abdominal diagnosis and Dangkisoo-san (Dangguixu-san) Herb-medication for the Traffic Accident Patients. J Oriental Rehabilitation Medicine. 2007;17(4):209-21.

\section{ORCID}

Jae Ryong Bae: https://orcid.org/0000-0003-1894-6091

Hun Mo Ahn: https://orcid.org/0000-0002-8320-7132

Ju Hee Roh: https://orcid.org/0000-0002-7899-6392

Min Gun Jo: https://orcid.org/0000-0003-4323-2699

Hye Ryeon Kim: https://orcid.org/0000-0002-9574-8657

Seon Hye Kim: https://orcid.org/0000-0002-8564-7457

Won Suk Sung: https://orcid.org/0000-0003-0585-9693

Eun Jung Kim: https://orcid.org/0000-0002-4547-9305 